\title{
Oral \& Dental Health Knowledge and Attitude among Nursing
}

\section{Students}

\author{
Leena Smadi ${ }^{*} \&$ Omayah S Nassar ${ }^{2}$ \\ ${ }^{1}$ Department of conservative dentistry, University of Jordan, Amman, Jordan \\ ${ }^{2}$ Department of Maternal and child health, Faculty of Nursing, University of Jordan, Amman, Jordan \\ *Leena Smadi, E-mail: alsomadi@yahoo.com
}

\begin{abstract}
Objectives: Investigate knowledge of oral health and oral diseases and attitudes about oral health care education among students of nursing, and willingness of students to introduce such training into their curriculum.

Methods: A cross sectional survey study was done on Jordan university nursing students using a questionnaire testing knowledge and attitude.

Results: 184 students completed questionnaires, for a response rate of $92 \%$. No special modules were for the students. Poor knowledge score was obtained with score of $46 \%$ in general oral health, $48 \%$ in pediatric dentistry questions, $43 \%$ in dentistry related to systemic diseases questions. $90 \%$ of students believe that oral health care is a high priority and consider treatment in the oral cavity as much important as treatment in other parts of the body with $80 \%$ of students think that it is necessary to obtain Oral health curriculum education with appropriate curriculum competence level assessment Approximately $85 \%$ would like to obtain more Oral health curriculum education and to implement Oral Health Curriculum activities during their training and career.

Conclusions: Oral and dental health knowledge level was poor among nursing students. There is a need for oral and dental health structured training to be incorporated in the curriculum of nursing students at university level.
\end{abstract}

\section{Keywords}

dental education, inter professional care, nursing curriculum, oral health

\section{Introduction}

Oral conditions, such as tooth decay, periodontal disease and oral cancer, are among the most common and wide spread diseases of humankind. They are generally related to the same preventable risk factors associated with over 100 non-communicable diseases. Yet, international attention to oral diseases does not match the high number of cases, nor the impact these diseases have on individuals, populations and society (FDI World Dental Federation).

Oral diseases are often hidden and invisible, or they are accepted as an unavoidable consequence of life 
and ageing. However, there is clear evidence that oral diseases are not inevitable, but can be reduced or prevented through simple and effective measures at all stages of the life course, both at the individual and population levels (Richard G).

Untreated tooth decay is now known to be the most prevalent of the 291 conditions studied between 1990 and 2010 within the frame of the international Global Burden of Disease Study (Marcenes, W. et al., 2013). This is the most authoritative estimation of global disease burden and serves as a basis for health policy planning and resource allocation. Severe periodontitis, which is estimated to affect between 5 and 20 percent of populations around the world, was found to be the sixth most common condition (Irfan et al., 2001). Oral cancer is among the 10 most common cancers in the world, and even more prevalent in South Asia, with numbers expected to rise due to increasing tobacco and alcohol (FDI World Dental Federation).

Historically, approaches to oral care have focused on individual curative care rather than on population-based preventive interventions. However, the financial and human resource costs of this approach are unaffordable for many countries, and unsustainable on a global scale (FDI World Dental Federation).

Prevention of oral disease and promotion of oral health can be directed towards individuals, communities or entire populations.

The integration of oral and general health should be the cornerstone of policy approaches to improve prevention and control of oral diseases. This is acknowledged in the Oral Health Action Plan adopted by the 60th World Health Assembly in 2007. This emphasizes "the intrinsic link between oral health, general health and quality of life" and identifies "the need to incorporate programs for promotion of oral health and prevention of oral diseases into programs for the integrated prevention and treatment of chronic diseases" (60th world health assembly resolution A16).

One of the main challenges toward this integration is the lack of interest, knowledge and education for non-dental health care providers (physicians, pharmacists, nurses) for oral and dental health. Oral health is not considered as one of nurses duties in practice (Clemmens). This might be a reflection of lack of basic oral health in curriculums of nursing education institutes (Institute of Medicine).

To improve health care providers' knowledge and attitudes, collaboration between regular health care and oral health care has to be included in health care education (Relf et al., 2009; Naidoo et al., 2007). A formal training of attending nurse students in the recognition prevention and safe management of oral diseases is required (Wårdh et al., 2000, 2008).

The aim of the study was to investigate knowledge of oral health and oral diseases and attitudes about oral health care among students of nursing. 


\section{Method}

A cross sectional study was done on Jordan university nursing students during the first semester of the academic year 2015-2016. The Faculty of Nursing (FON) was established in 1972. It was the first program in Jordan that offered a Baccalaureate Degree in Nursing and was also the first to establish a nursing education program at the graduate level. The total number of students at the faculty is around 800 students. The target group for this survey was a final year students at faculty of nursing comprised of 200 student.

The survey was conducted using a questionnaire probing on specific socio-demographic characteristics, knowledge and practices on selected oral health issues, the survey was voluntary, and responses were anonymous. Ethical clearance was sought from the ethical committee of the Deanery of Academic Research of the university.

The questioner assessed two domains. The first was oral and dental health knowledge, and the second looked at student's attitude toward subjects of oral and dental health.

The questionnaire was administered at the same time to all of the participants in the respective class rooms. Authors were available in those class rooms to clarify on any question arising in regard to the questionnaire.

The questionnaire of the study was developed based on previous validated surveys (Rabiei et al., 2014; dela Cruz et al., 2004; Giuseppe et al., 2006; Parakash et al., 2006) with minor modifications. The questionnaire was assessed for content validity by experts in dental public health for relevancy and clearness.

The questionnaire was divided into Demographic information related questions (2 questions), Curriculum related questions (4 questions), which included questions on whether the curriculum includes oral health defined as everything that concerns the mouth area; the teeth, the gums, the tongue, the cheeks, etc. In addition to oral hygiene, oral diseases and eating habits, Personal attitudes concerning oral health care (4 questions), and Oral health knowledge related questions (14 questions), Paediatric dentistry related questions (21 questions), Dentistry related systemic questions (11 questions), Attitudes and willingness (4 questions). Personal attitudes concerning oral health care and willingness were measured on the 5-point Likert scale (Likert) with response alternatives ranging from "strongly agree" to "strongly disagree" including "don't know" knowledge questions were multiple choice questions.

To obtain the genuine responses, the anonymity of the respondents was ensured.

We dichotomized answers to the knowledge and attitude questions with a score of one for correct/willing/positive answers, and 0 for false/unwilling/negative and don't know answers. Statistical analysis was performed using SPSS 20.0 for Windows (SPSS Inc., Chicago, IL, USA). Student's t tests for independent variables, the level of significance was set at $\mathrm{p}<0.05$. 


\section{Result}

\subsection{Response}

184 students completed questionnaires, for a response rate of $92 \% .75 \%$ were females reflecting the same students gender ratio in the faculty ( $77.4 \%$ females), median age was 21.3 years, and all students were in their final year of studying.

\subsection{Curriculum Related Questions}

None of the responding students studied a special oral or dental health modulus, all students studied oral health in their curriculum as part of other modules.

\subsection{Knowledge Domain}

Oral health knowledge score was 6.5 out of 14, Paediatric dentistry knowledge score was 9.99 out of 21, Dentistry related systemic knowledge score was 4.78 out of 11 , total knowledge score was 21.27 out of 46 (Table 1).

When these scores converted to percentages for better comparison, students scored $46 \%$ in general oral health related questions, $48 \%$ in paediatrics dentistry questions, $43 \%$ in dentistry related to systemic diseases questions, with over all knowledge score of $46 \%$ in all subjects (Figure 1).

More detailed information about level of knowledge related to studied domains of oral and dental health are presented in Tables 2, 3, 4 and 5.

\subsection{Attitude and Willingness Domain}

More than $90 \%$ of students believe that oral health care is a high priority and consider treatment in the oral cavity as much important as treatment in other parts of the body and also consider oral health as an integral part of general health. Only $86 \%$ of students consider regular visit to the dentist necessary (Figure 2).

More than $80 \%$ of students think that it is necessary to obtain Oral health curriculum education and it is necessary to assess Oral health curriculum competence level (to pass oral health exam). Approximately $85 \%$ would like to obtain more Oral health curriculum education and to implement Oral Health Curriculum activities during their training and career (Figure 2).

\section{Table 1. Knowledge Domain}

\begin{tabular}{llllll}
\hline & Number of questions & Average score & Min & Max & SD \\
\hline Oral health knowledge related questions & 14 & 6.5 & 2 & 11 & 4.5 \\
Paediatric dentistry related questions & 21 & 9.99 & 1 & 16 & 7.5 \\
Dentistry related systemic questions & 11 & 4.78 & 0 & 10 & 5 \\
Total score & 46 & 21.27 & 4 & 35 & 15.5 \\
\hline
\end{tabular}


Table 2. Oral Health Knowledge Related Questions

\begin{tabular}{ll}
\hline Oral health knowledge related questions & $C A \%{ }^{*}$ \\
\hline What is dental plaque? & 38 \\
What is caries? & 68 \\
What causes caries? & 73 \\
What is gingivitis? & 19 \\
What causes gingivitis? & 18 \\
What is periodontitis? & 63 \\
What causes periodontitis? & 65 \\
What is the reason for brushing the teeth? & 61 \\
What is the purpose of fluorides? & 40 \\
The acid produced by oral bacteria after a patient eats sugar is buffered by the saliva. \\
A tooth avulsed due to trauma can be re-implanted into the tooth socket. & 45 \\
Artificial teeth can perfectly replace the function of natural teeth. & 41 \\
Soft drinks can cause erosion of dental enamel which is the hardest tissue in the human body. \\
Loss of teeth during old age is a natural phenomenon. Neither the dentist nor the patient can prevent tooth loss.
\end{tabular}

*CA: percentages of correct answer.

\section{Table 3. Paediatric Dentistry Related Questions}

\begin{tabular}{ll}
\hline Paediatric dentistry related questions & $C A \% *$ \\
\hline Mean age of children when first permanent teeth erupt in the mouth. & 64 \\
Mean age of infant when the first deciduous teeth erupt in the mouth. & 56 \\
Which permanent tooth erupt first. & 14 \\
Proper maintenance of deciduous dentition is as important as the permanent dentition. & 52 \\
Prenatal care can affect the dental status of the baby later in life. & 61 \\
Micro-organisms that cause dental caries are transmitted mainly from the mother to the child. & 33 \\
Frequency of sugar consumption has a greater role in producing caries than does the total amount of sugar & 65 \\
consumed. & 71 \\
Para-functional habits like thumb sucking, lip biting, lip sucking and nail biting are very common among \\
children. These habits need to be curbed as they affect oro-facial structures. \\
Alignment of teeth is done both for functional as well as aesthetic purpose. \\
Mouth-guards are useful in preventing sport-related injuries/trauma.
\end{tabular}

*CA: percentages of correct answer. 
Table 4. Paediatric Dentistry Related Questions

Paediatric dentistry related questions

Cleft palate and cleft lip are developmental defects. Proper surgical and prosthetic treatment is available 72 that will enable patients with Cleft lip/Cleft palate to lead a normal life.

Fluoridated tooth paste should not be used for children under 3 years old.

Teeth cleaning and brushing should be started from 2-3 years old.

Fluoride varnish on those under age 5 years causes fluorosis and poisoning.

Sealants are effective in the prevention of pit and fissure caries.

Dental decay of deciduous teeth surfaces in pre-school children can predict having decay in the future. 56

Mouth breathing is a risk factor for dento-alveolar malformation.

Using fluoride tooth paste is more important than the brushing technique for preventing caries.

Using xylitol in the form of gum or snacks reduces the level of bacteria which causes decay.

Mouth rinsing with a high amount of water after tooth brushing with fluoride containing tooth paste is 21

effective in preventing decay.

The first signs of decay are white spots or lines on teeth surfaces.

*CA: percentages of correct answer.

Table 5. Dentistry Related Systemic Questions

Dentistry related systemic questions

Certain systemic diseases can manifest in the oral cavity.

Salivary Biomarkers can be used for detection of certain Systemic Diseases.

Oral diseases have an implication on certain systemic diseases/conditions like cardiovascular diseases, 54 Pregnancy, low birth weight babies, etc.

The best time to refer a pregnant woman for dental procedure is in: 30

Some antihypertensive drugs may increase the risk for dental caries.

Periodontal diseases of the pregnant mother can cause low birth weight.

Periodontal diseases can cause problems in diabetic control.

Radiotherapy increase the risk of dental caries.

Chemotherapy increase the risk of dental caries.

Periodontal diseases can cause cardiovascular disease.

Lesions on the oral cavity which needs biopsy. 30

*CA: percentages of correct answer. 


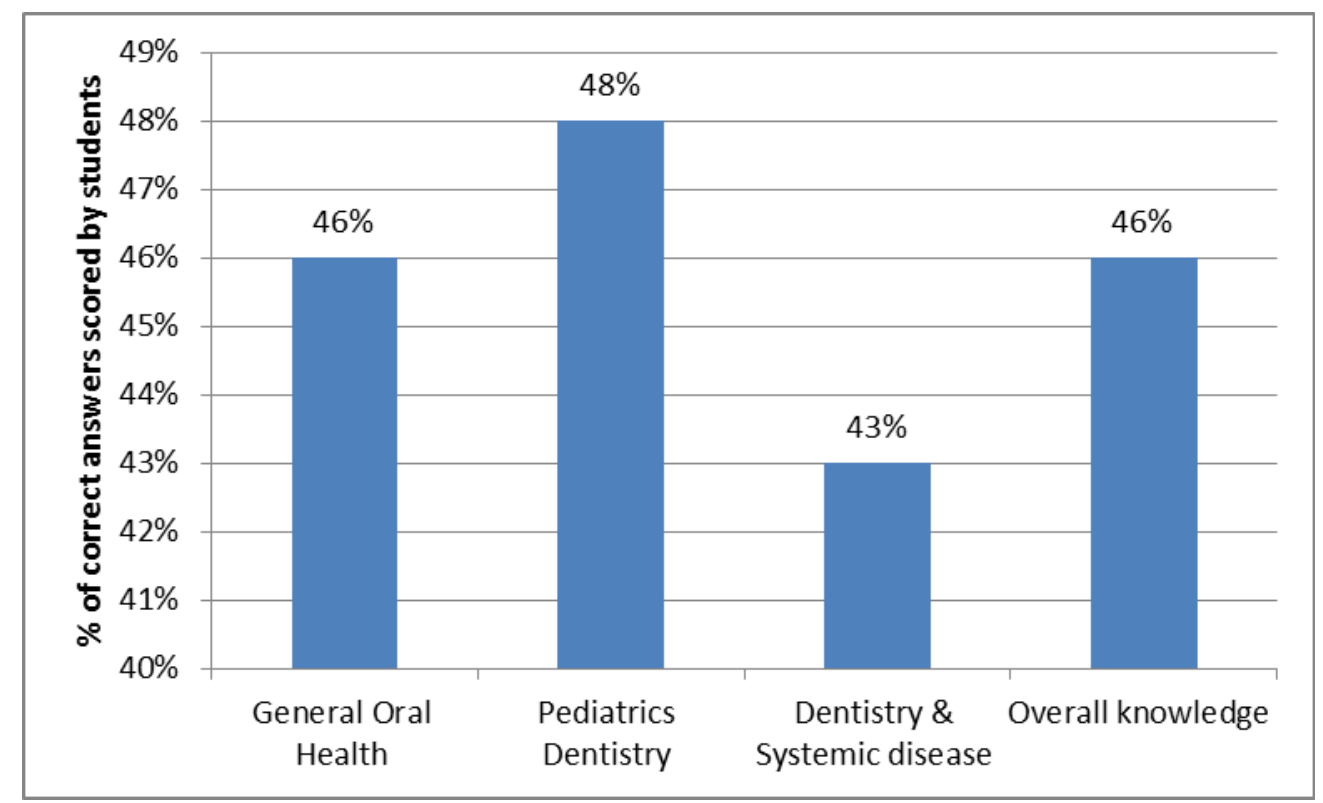

Figure 1. Knowledge Level Related to Oral and Dental Health Care among Nursing Students

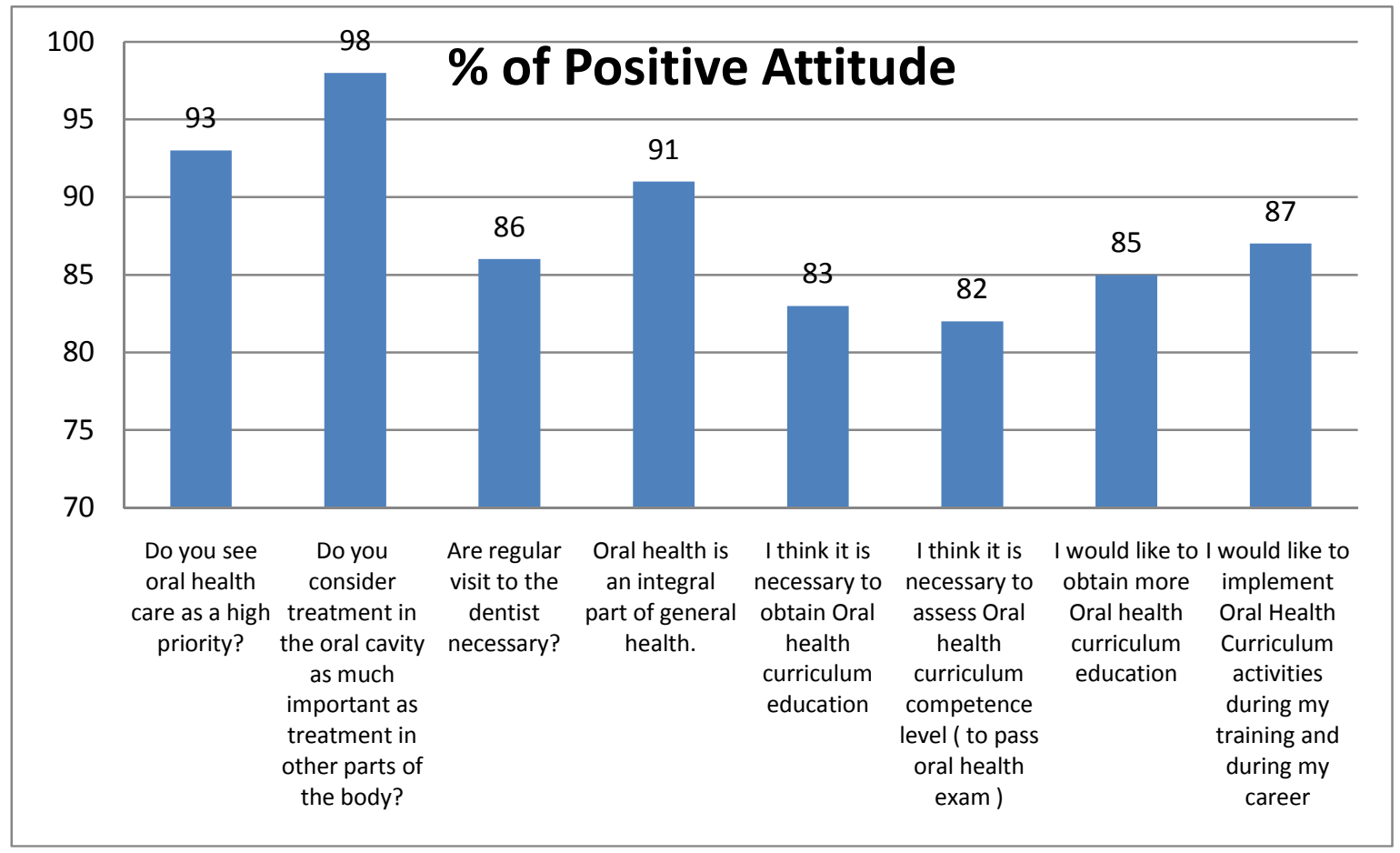

Figure 2. Positive Attitude toward Oral and Dental Health Care and Willingness for Further Training among Nursing Students 


\section{Discussion}

Nurses, including registered nurses, licensed practical nurses, make up the largest proportion of health care professionals. Registered nurses and advanced practice nurses for example represented roughly 37 percent of all healthcare professionals in United States of America, while LPNs and LVNs represented an additional nine percent (Bureau of Labor Statistics), during the year 2014 there are 27.6 RN per 100000 population in 2014 and 13 Associate Nurse per 100000 in Jordan compared to only 10.5 dentists per 100000 (Jordanian Ministry of Health) this group of health care professionals encounters far more underserved and needed population who needs oral and dental care than dental care professionals (Institute of Medicine). Therefore, it is essential that these health care providers are familiar with the various risk factors to manage oral care and make appropriate referrals and intervention decisions as recommended by the Institute of Medicine (IOM) report. University education is the first step in introducing oral health knowledge and ability of integration approach to curricular change that embeds oral health and knowledge subjects into existing nursing professional education is of utmost important to reach such goals.

Our results showed lack of such integration of oral health and dental education into the curriculum of nursing faculty at university of Jordan with $100 \%$ response by the students that none of them received structured training or special module. This fact is not an exception among nursing education programs, for example in survey of 1,000 basic nursing education programs in USA, over half of the schools reported little curriculum content regarding health promotion for oral health and dental care; this was attributed to lack of faculty time, interest and expertise (Smeltzer et al., 2005; Kraus et al., 2002; Spielman et al., 2005).

This lack of structured education and training was evident by ability of the students to show enough knowledge to answer the questions related to dental and oral science, with less than $50 \%$ of the students got correct answers, the ability to show satisfactory knowledge in our group was less than that reported by Al-Batayneh et al. (2014) and MOHD-DOM et al. (2009) who showed better level in knowledge among 78 nursing students in their study, nevertheless nursing students showed statically significant lower level of knowledge when compared to either pharmacy students or medical students, this difference between our results and the other two studies might be explained by more specific questions related to dental diseases in our study compared to more general oral health questions in the two studies. However direct comparison of knowledge level was difficult to ascertain with many other studies (Al-Ansari et al., 2003; Skelton et al., 2002) due to the different contents of knowledge questions in questionnaires used.

Detailed analysis of knowledge contents among our study populations showed that $62 \%$ of the students do not know what dental plaque is and less than $20 \%$ know its role as a cause of gingivitis or periodontitis. $37 \%$ of students correlate smoking as a direct cause of gingivitis. Less than $50 \%$ of students know that a tooth avulsed due to trauma can be re implanted into the tooth socket and soft drinks can cause dental erosion. $70 \%$ of students consider loss of teeth during old age as a natural 
phenomenon, and neither the dentist nor the patient can prevent tooth loss.

In general, nursing students showed limited dental knowledge related to oral health which will makes them incapable of providing the appropriate care by early detection and prevention of oral disease. This lack of ability to provide such care was showed in different studies were lack of knowledge related to oral health was the role among non-dental health care providers (Priya et al., 2008; Wu et al., 2006).

In systemic health domain approximately third of the students know the best time to refer a pregnant woman for dental procedure and that periodontal diseases of the pregnant mother can cause low birth weight. The same applies to the correlation between periodontal disease and diabetic control or cardiovascular disease. About half of students are aware of role of radiotherapy or chemotherapy as a risk factor of dental decay, the percentage was much lower (34\%) for the role of some antihypertensive drugs as a risk factor of dental decay. Only $30 \%$ answered correctly which lesions of the oral cavity needs biopsy. These results indicate that training to recognize oral problems, oral lesions, or oral squeal of chronic systemic conditions and the medications to treat these conditions is limited. The lack of this form of knowledge is not limited to our study population, but it was demonstrated in other studies (Ettinger), this will reflect on safe health care provided by nurses to patients treated for non-dental problems, mainly systemic diseases. Thai et al. (1997) for example concluded in his study that the nurse's assessments identified few oral health problems and that the problems identified did not translate into dental treatment. A study by Blank et al. (1996) found that more experienced nurses were able to identify broken or carious teeth nearly 85 percent of the time among nursing home residents. However, regardless of the nurse's experience level, they were less able to identify soft tissue lesions. Taken together, the potential for misidentification, underreporting of oral health problems and proper referral is difficult to ignore, which makes professional bodies like the American College of Nurse-Midwives to raise the alert about the role of nurses in proper management and referrals for patients who needs extra care for oral diseases, lack of knowledge and low expectations about oral health and its value influence care-seeking behavior and can result in care being deferred or neglected entirely (Michael et al., 2002).

The same trend of lack of knowledge was found when pediatrics related oral and dental health questions were asked with less than $40 \%$ of students do not know the mean age of children when first permanent teeth erupt in the mouth or when the first deciduous teeth erupt in the infant's mouth. Only $14 \%$ of students know which permanent tooth erupt first. About third of the students know that micro organisms that cause dental caries can be transmitted from the mother to the child.

Approximately $70 \%$ of students answered that Fluoridated tooth paste should not be used for children under 3 years old and that teeth cleaning and brushing should be started from 2-3 years old and that fluoride varnish on those under age 5 years causes fluorosis and poisoning, $80 \%$ of students answered that mouth rinsing with a high amount of water after tooth brushing with fluoride containing tooth paste is effective in preventing decay. Only $40 \%$ of students know that fissure sealants are effective in the prevention of pit and fissure caries. Approximately half of the students know that the first signs of 
decay are white spots or lines on teeth surfaces.

These results were better than those reported by Sepideh et al. (2014) in their study with only 19\% level of knowledge, similar results of poor knowledge was also demonstrated by Mohamed and Barnes. High proportion of students showed positive attitude for oral health subjects, with $98 \%$ of them showed positive attitude toward integration of oral health as part of general health, $93 \%$ agree that oral health issues are of utmost priority and $91 \%$ believe that oral health is important for general health, these results agree with other studies which examined the attitude among working nurses (McKinley et al., 2003; Wu et al., 2006; Baseer et al., 2012; Kaur et al., 2015; Mohebbi et al., 2012).

This positive attitude toward oral health did reflect in the direction but not magnitude of welling of introducing oral health subjects to the curricula of nursing schools , were only $83 \%$ think it is necessary to obtain Oral health curriculum education, $82 \%$ believe it is necessary to assess Oral health curriculum competence level or to pass oral health exam, $85 \%$ would like to obtain more Oral health curriculum education and $87 \%$ would like to implement Oral Health Curriculum activities during their training and during their career.

Same attitude toward introducing oral health teaching among university teaching was showed by McKinley et al. (2003), Mohamed (2015) Rabiei (2014), and Barnes (2015).

This positive attitude and willingness should be met by efforts to introduce oral health courses into the curriculum of undergraduate nursing teaching. Such courses were examined both on undergraduate or postgraduate training and proved efficacy (Hahn et al., 2012) and showed for example that incorporating an interdisciplinary, multifaceted oral health educational curriculum in a pediatric nurse regarding the inclusion of oral health assessments, consultations, preventive treatments and referral (Golinveaux et al., 2013).

In conclusion, oral and dental health knowledge level was poor among nursing students at Faculty of Nursing/Jordan University. This is due to failure of integrating oral and dental health care competence training into the curriculum of the faculty, and lacking of structured special modules of such training. This will reflect negatively on the level of care such students will deliver once they start their career, despite the fact that majority of them recognize the importance of such training, and their willingness to obtain structured training with proper assessment for competences in oral health subjects. We therefore recommend that oral dental health knowledge and structured training is incorporated in the curriculum of nursing students at university level. Such integration will need more post intervention studies and evaluation to test the effectiveness of such changes in the curriculum.

\section{References}

60th world health assembly resolution A16. (2007). World Health Organization Oral health: Action plan for promotion and integrated disease prevention. Geneva, WHO.

Al-Ansari, J., Honkala, E., \& Honkala, S. (2003). Oral health knowledge and behaviour among male health sciences college students in Kuwait. BMC Oral Health. 
Al-Batayneh, O. B. et al. (2014). Oral Health Knowledge and Practices among Diverse University Students with Access to Free Dental Care: A Cross-Sectional Study. Open Journal of Stomatology, 4, 135-142.

American College of Nurse-Midwives. (2014). Dental Care in Pregnancy. Retrieved from http://www.midwife.org/acnm/files/ccLibraryFiles/Filename/000000003904/Dental_Care_Pregna ncy.pdf

Baseer, M. A. et al. (2012). Oral health knowledge, attitude and practices among health professionals in King Fahad Medical City, Riyadh. Dent Res J (Isfahan), 9, 386-392.

Blank, L. et al. (1996). The effect of nurses' background on performance of nursing home residents' oral health assessments pre-and post-training. Spec Care Dentist, 16, 65-70.

Bureau of Labor Statistics "Occupation Profiles". (2014). Occupational Employment Statistics, U.S. Department of Labor. Retrieved April 1, 2014, from http://www.bls.gov/oes/current/oes_stru.htm\#29-0000

Clemmens, D. A., \& Kerr, A. R. (2008). Improving oral health in women: Nurses' call to action. The American Journal of Maternal/Child Nursing, 33(1), 10-14.

dela Cruz, G. G., Rozier, R. G., \& Slade, G. (2004). Dental screening and referral of young children by pediatric primary care providers, 114, 642-652.

Di Giuseppe, G., Nobile, C. G., Marinelli, A., \& Angelillo, I. F. (2006). Knowledge, attitude and practices of Pediatricians regarding the prevention of oral diseases in Italy. BMC Public Health, 6(176). http://dx.doi.org/10.1186/1471-2458-6-176

Ettinger, R. (1992). Oral care for the homebound and institutionalized. In B. Baum (Ed.), Clinics in geriatric medicine: Oral and dental problems.

FDI World Dental Federation. (2015). The Challenge of Oral Disease-A call for global action. The Oral Health Atlas (2nd ed.). Geneva.

Golinveaux et al. (2013). Oral Health Education for Pediatric Nurse Practitioner Students. Journal of Dental Education, 77(5), 581-590.

Hahn et al. (2012). Infusing Oral Health Care into Nursing Curriculum: Addressing Preventive Health in Aging and Disability. Nursing Research and Practice, 2012.

Institute of Medicine. (2011). Improving Access to Oral Health Care for Vulnerable and Underserved Populations. The National Academies Press, Washington, DC, USA. Retrieved from http://www.iom.edu/Reports/2011/Improving-Access-to-Oral-Health-Care-for-Vulnerable-and-Un derserved-Populations.aspx

Irfan, U. M., Dawson, D. V., \& Bissada, N. F. (2001). Epidemiology of periodontal disease: A review and clinical perspectives. $J$ Int Acad Periodontol, 3, 14-21.

Jordanian Ministry of Health web site. (2016). Retrieved from http://www.moh.gov.jo/AR/Pages/mainind.aspx?ind=http\%3a//apps.moh.gov.jo/reports/headermai n.jsp?firstjsp=manpowermain\&lang_parameter $=$ english 
Kaur, S., \& Ahluwalia, S. S. (2015). Oral Health Knowledge, Attitude and Practices amongst Health Professionals in Ludhiana, India. Dentistry, 5, 315.

Kraus, M. C., Connick, \& Morgan, C. (2002). Interdisciplinary partners: nursing and dental hygiene. The Journal of Nursing Education, 41(12), 535-536.

Marcenes, W., Kassebaum, N. J., Bernabe, E., Flaxman, A., Naghavi, M., Lopez, A., \& Murray, C. J. (1990-2011). Global Burden of Oral Conditions in 1990-2010: A Systematic Analysis. J Dent Res, 92, 592-597.

McKinley, V. A., Thomson, W. M., \& Ayers, K. M. (2003). A qualitative study of oral health knowledge and attitudes among staff caring for older people in Dunedin long-term care facilities. $N Z$ Dent $J$, 99(4), 98-103.

Michael, J. et al. (2002). Dental Considerations for the Frail Elderly. Spec Care Dentist, 22(3), 40S-55S.

Mohamed, N., \& Barnes, J. (2015). Knowledge, Attitudes and Practices (Kap) Regarding Early Childhood Caries among Nurses Working in a Low Socio-Economic Area. Arch Community Med Public Health, 1(1), 1-5.

Mohd-Dom, S., \& Aidin, Z. (2009). Dental Knowledge and Self-reported Oral Care Practices Among Medical, Pharmacy and Nursing Students. Jurnal Sains Kesihatan Malaysia, 7(1), 13-23.

Mohebbi, S. Z., Virtanen, J. I., Vahid-Golpayegani, M. B., \& Vehkalahti, M. M. (2009). Trial of Effectiveness of Educational Intervention in Primary Health Care on Early Childhood Caries. Caries Res, 43, 110-118.

Naidoo, S., \& Myburgh, N. (2007). Nutrition, oral health and the young child. Maternal \& Child Nutrition, 10(3), 312-321.

Oral Health Worldwide. (2014). A report by FDI World Dental Federation. Retrieved from http://www.worldoralhealthday.com/wpontent/uploads/2014/03/FDIWhitePaper_OralHealthWorld wide.pdf

Prakash, P., Lawrence, H. P., Harvey, B., Maclsaac, W. J., Limeback, H., \& Leake, J. L. (2006). Early childhood caries and infant oral health: Pediatricians' knowledge, practices and training. Paediatr Child Health, 11, 151-157.

Priya, S., Madan, Kumar, P. D., \& Ramachandran, S. (2008). Knowledge and attitudes of pharmacists regarding oral health care and oral hygiene products in Chennai city. Indian J. Dent. Res, 19, 104-108.

Rabiei et al. (2014). Primary care nurses' awareness of and willingness to perform children's oral health care. BMC Oral Health, 14(26).

Rabiei, S., Mohebbi, S. Z., Patja, K., \& Virtanen, J. I. (2012). Physicians' knowledge of and adherence to improving oral health. BMC Public Health, 12(855). http://dx.doi.org/10.1186/1471-2458-12-855

Relf, M. V., Laverriere, C., Devlin, C., \& Salerno, T. (2009). Ethical beliefs related to HIV and AIDS 
among nursing students in South Africa and the United States: A cross-sectional analysis. International Journal of Nursing Studies, 1(46), 1448-1456.

Rensis, L. (1932). A Technique for the Measurement of Attitudes. Archive of Psychology, 22, 5-55.

Skelton, J., Smith, T. A., Betz, W. T., Heaton, L. J., \& Lillich, T. T. (2002). Improving the oral health knowledge of osteopathic medical students. J. Dent. Educ, 66(11), 1289-1296.

Smeltzer, S. C. M. A., Dolen, G., Robinson-Smith, \& Zimmerman, V. (2005). "Integration of disability-related content in nursing curricula”. Nursing Education Perspectives, 26(4), 210-216.

Spielman, T., Fulmer, E. S., Eisenberg, \& Alfano, M. C. (2005). Dentistry, nursing, and medicine: A comparison of core competencies. Journal of Dental Education, 69(11), 1257-1271.

Thai, P. H., Shuman, S. K., \& Davidson, G. B. (1997). Nurses' dental assessments and subsequent care in Minnesota nursing homes. Spec Care Dentist, 17, 13-18.

Walid, E. I., Nasir, F., \& Naidoo, S. (2004). Oral health knowledge, attitudes and behaviour among nursing staff in Lesotho. SADJ, 59(7), 288, 290, 292.

Wårdh, I., Andersson, L., \& Sörensen, S. (2008). Staff attitudes to oral health care. A comparative study of registered nurses, nursing assistants and home care aides. Gerodontology, 14(1), 28-32.

Wårdh, I., Hallberg, L., Berggren, U., Andersson, L., \& Sörensen, S. (2000). Oral health care-Low priority in nursing. Scandinavian Journal of Caring Sciences, 14(2), 137-142.

Watt, R. (2005). Strategies and approaches in oral disease prevention and health promotion. Bulletin of the World Health Organization, 83(9).

Wu, I. M., King, N. M., Tsai, J. S. J., \& Wong, H. M. (2006). The dental knowledge and attitudes of medical practitioners and caregivers of pre-school children in Macau. Hong Kong. J. Pediatr, 11, 133-139. 